\title{
Assessment of Muscle Overactivity and Spasticity with Dynamic Polyelectromyography and Motion Analysis
}

\author{
Alberto Esquenazi ${ }^{*}$, , Matteo Cioni $^{2}$ and Nathaniel H. Mayer ${ }^{1}$ \\ ${ }^{I}$ Gait and Motion Analysis Laboratory, MossRehab, Elkins Park, PA, USA \\ ${ }^{2}$ Gait and Motion Analysis Laboratory, School of Medicine, University of Catania, Italy
}

\begin{abstract}
Damage of the central nervous system often produces an upper motoneuron (UMN) syndrome. Characteristics of the UMS include the presence of positive and negative signs. Positive signs are increased phasic and tonic stretch reflexes, clonus, co-contraction, released flexor reflexes, spastic dystonia, and associated reactions, whereas negative signs signify the loss or impairment of voluntary movement assembly and production. Muscle overactivity seems to be a more suitable term than spasticity, because it evokes more an image of dynamic muscle contraction, which is the general hallmark of all positive signs of UMS. Some of the main patterns of UMN dysfunction include: equinovarus foot, hyperextended great toe, stiff knee, adducted (scissoring) thighs, and flexed hip. The dynamic poly-EMG recordings of trunk and lower limb muscles in combination with the clinical examination provide a more detailed understanding of the genesis of these abnormal patterns in the UMN.
\end{abstract}

Keywords: UMN syndrome, muscle overactivity, spasticity, poly-EMG.

\section{UPPER MOTONEURON SYNDROME}

Spasticity is a term that clinicians use with familiarity but that can be interpreted in many ways by different individuals. Spasticity is just one of the many positive signs of the upper motor neuron (UMN) syndrome; yet, under the heading of spasticity, clinicians often group together all positive signs and sometimes inadvertently may include even some of the negative signs.

The issue of terminology is more than one of semantics as the appropriate phenomenological identification will help identify the best possible treatment intervention. Many of these frequently misidentified phenomena are characteristic of the broader term UMN syndrome, a condition that has classically been partitioned into a neurological syndrome with positive and negative signs. Negative signs signify the loss or impairment of voluntary movement assembly and production, a kind of muscle underactivity that, in effect, can be described as a phenomena of absence and include weakness and loss of dexterity while increased phasic and tonic stretch reflexes, clonus, co-contraction, released flexor reflexes, spastic dystonia, and associated reactions are viewed as phenomena of presence, a kind of muscle overactivity whose clinical manifestations are often untimely and inopportune [1].

The treatment of co-contraction, a phenomenon likely to be of supraspinal origin, will differ from treatment of clonus, a phenomenon of the segmental stretch reflex loop. Clinicians are encouraged to utilize a concise, descriptive, utilitarian term that captures the essence of the positive UMN phenomena, muscle overactivity as proposed by

*Address correspondence to this author at the Gait and Motion Analysis Laboratory, MossRehab, Elkins Park, PA, USA; Tel: 1215663 6676; Fax: 1215663 6686; E-mail: aesquena@einstein.edu
Table 1. Some of the Phenomena Affecting the Balance Between Agonist-Antagonist Muscles

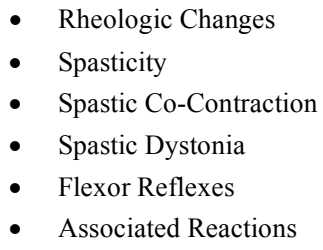

Mayer and Esquenazi may be a more suitable term than spasticity, especially because the term evokes an image of dynamic muscle contraction, the general hallmark of all positive signs of UMN [2].

Whatever else is meant by the term spasticity, it has classically meant increased excitability of skeletal muscle stretch reflexes, both phasic and tonic, that are typically present in most patients with a UMN lesion. After a UMN lesion, a net loss of inhibition impairs direct descending control over motor neurons. There is also a loss of inhibitory control over interneuronal pathways of the cord that ordinarily regulate segmental spinal reflexes, including stretch reflexes, especially those concerned with antigravity muscles. Lance characterized spasticity as an increase in velocity-dependent tonic stretch reflexes with exaggerated tendon jerks [3]. Generally, the term phasic implies time varying, whereas tonic has a time-invariant quality, though time scales are always relative.

However, the literature's use of the terms phasic and tonic can be confusing because some authors refer to an input stimulus as either phasic or tonic, whereas others characterize the output response as either phasic or tonic. In the consensus definition of Lance [3], tonic stretch reflexes referred to the output response of a muscle group that was 
stretched at different velocities. "Exaggerated tendon jerks" were examples of phasic stretch reflexes. In routine practice at the bedside, the two ways of assessing phasic and tonic stretch reflexes are tendon taps and passive stretch of a muscle group at different rates of stretch.

Though phenomena of presence are a common source of clinical concern and frequent targets of intervention, phenomena of absence such as weakness may be more functionally disabling and resistant to treatment. The clinical picture is made more complex by another phenomenon of presence that has not been classically positioned among the positive signs, namely contracture, physical changes in the rheologic properties of muscle. These rheologic changes in viscoelastic and plastic properties of muscle and other soft tissues, especially when they mature into a contracture, are well recognized as a major source of disability for patients with a UMN syndrome. Positive and negative phenomena as such vary in their pathophysiology and appropriate identification will likely have an impact on treatment selection.
From a pathological point of view, spasticity is a velocity-dependent phenomena. In fact, when slowly moving the elbow of a subject with a spastic limb we can see a small amount of EMG activity in brachioradialis (Fig. 1) by contrast when the speed of movement is increased one can observe a corresponding increase in the EMG activity, and when the movement is very fast a great increase in the EMG activity is present. In contrast normal individuals exhibit very small EMG changes in response to velocity variations.

Co-contraction is the simultaneous activation of antagonist muscle alongside voluntary contraction of agonist muscles, in absence of a triggering stretch reflex. Cocontraction is a very inefficient energy muscle contraction. Physiologically co-contraction is seen in very young children to stabilize the trunk and to control the displacement of the center of gravity. This physiological phenomenon disappears with maturation of the central nervous system along with the development of other more efficient mechanisms such as postural reactions. The mechanism of co-contraction reappears as part of the UMN syndrome. In Fig. (2), the EMG activity of a patient with spastic co-contraction who

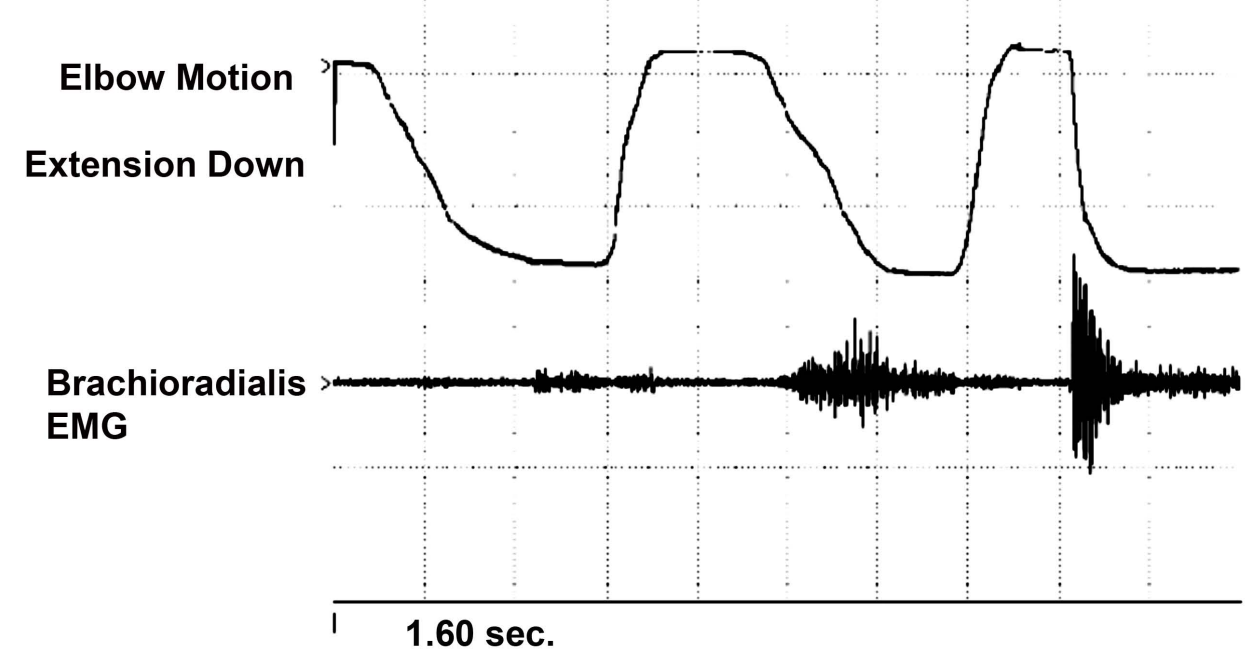

Fig. (1). Electromyographic responses of muscle brachioradialis to slow, moderate and rapid passive stretches of elbow, in a patient with cerebral palsy. It can be seen the spastic increase of electromyographic response to the rapid passive stretching.

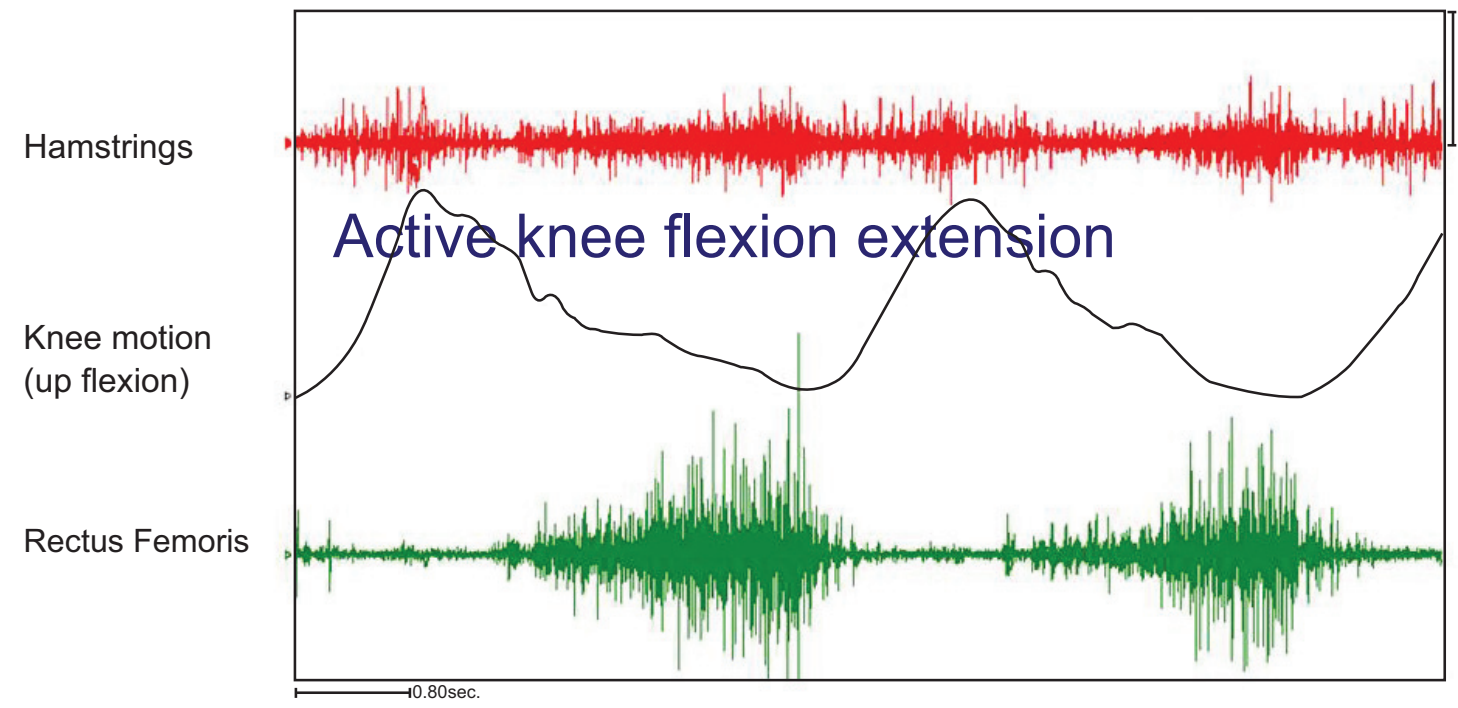

Fig. (2). Coactivation phenomenon between hamstrings and rectus femoris during active knee motion. 
was instructed to voluntarily move his leg from flexion to extension is depicted. This image illustrates knee flexion with activation of rectus femoris (knee extensors) with cocontraction of hamstrings (knee flexors), which reduces the fluidity of repeated movement of the knee in to extension.

Spastic dystonia In the absence of voluntary effort or passive stretch, UMNS patients with demonstrable tonic muscle activity underlying a persistent posture have been called 'dystonic', consistent with Denny-Brown's original construct. Dystonic activity is believed to result from an abnormal pattern of a supra-spinal involuntary drive. UMNS patients with dystonic activity also appear to have stretch sensitivity and, hence, have been described as having "spastic dystonia". Stretch sensitivity in UMNS patients can result not only in an increase but also a lessening of dystonic activity, especially true after prolonged stretch, a finding that has implications for therapeutic exercise and patient selfmanagement. Spastic dystonia can be seen in children with cerebral palsy and other neurological diseases. In Fig. (3) are depicted EMG recordings from the upper limb of a patient with spastic dystonia. The patient is at rest, while standing with the elbow flexed, but not due to a voluntary muscle contraction.

The enhaced nociceptice and cutaneous-motor reflexes are mediated by afferents from skin, subcutaneous, perimuscular or other tissues that respond to touch, pressure, temperature and pain. The response to the presentation of these reflexes is represented by a startle reaction, flexor spasms, extensor spasms and the Babinski sign. These reflexes are clinically triggered by touching the patient or by a sudden noise. Associated reactions are also called synkinesias. Walshe first described associated reactions as "released postural reactions deprived of voluntary control in 1923 [4].

"Synkinesis" is a term used by Bourbonnais more recently. An associated reaction is linked (or "associated") with a voluntary movement made in another limb [5].

It may be due to disinhibited spread of voluntary motor activity into the affected UMN limb and its intensity may depend on the intensity of the voluntary effort. Dewald and Rymer thought that impaired descending supraspinal commands generated associated reactions [6]. Clonus is a phenomenon that we often see both in infants and patients with CP. Clonus can be recorded by electromyography as a signal with a frequency of $5-7 \mathrm{~Hz}$, after stretching the patellar or Achilles tendons.

The patterns of limb dysfunction in UMN syndrome have an impact on the core priorities of limb usage for gait or functional use. A number of muscles typically cross major joints of the limbs, and identifying the actual muscles that contribute dynamically and statically to a UMN syndrome deformity is an important key to clinical management of the resulting gait or upper limb impairment and disability.

\section{Table 2. Patterns of Dysfunction in the UMN Syndrome} Affecting Walking

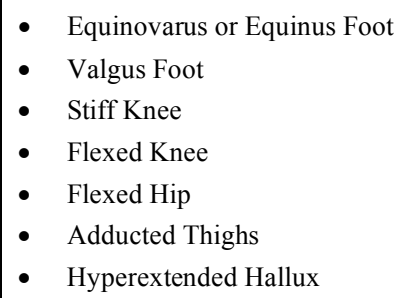

Clinical evaluation is useful to the analysis of impaired movement, but gait and motor assessment laboratory evaluation using dynamic electromyography (EMG) is often necessary to identify the particular contributions of offending muscles with confidence. The correct selection of target muscles that contribute to any one pattern of dysfunction may serve as a rational basis for interventions that focus on specific muscles for therapy.

More than fifty years ago, Nikolai A. Bernstein suggested that a basic problem of motor control relates to overcoming redundant degrees of freedom in our multijointed skeletal system that allow us to interact with the three dimensional world in which we live. Others in his tradition discovered the coordinative rules or priorities used by the brain to constrain redundant degrees of freedom when performing motor tasks. Commonly, there are multiple agonists and antagonists for virtually any movement direction. To match a required joint torque even across a
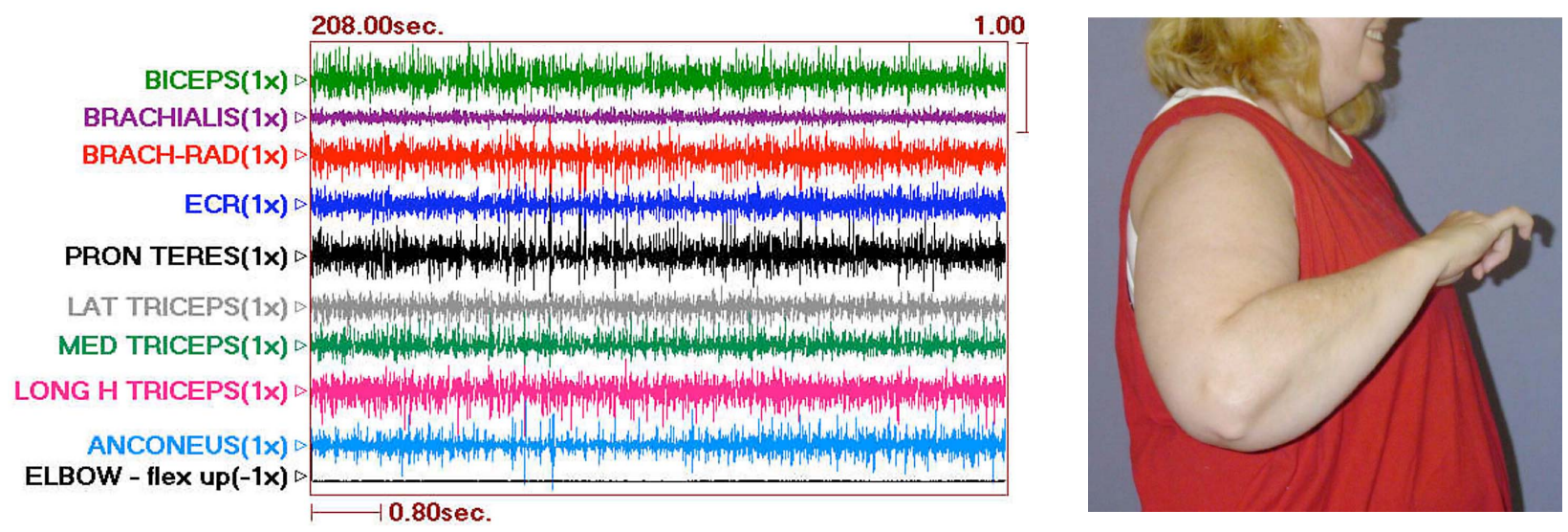

Fig. (3). This figure shows the electromyographic recordings from 9 muscles (biceps, brachialis, extensor carpi radialis, extensor carpi radialis, pronator teres, triceps lateral head, triceps medial head, triceps long head, anconeus ) of a patient with a flexed elbow posture, at rest. It can be noted that all muscles recorded are hyperactive. 
single joint, the question regarding which muscles should be activated and at what levels of activity is likely to have a very variable answer without a unique solution.

For a given patient, however, there may be a unique solution. As an example the equinovarus foot deformity may be solely attributable to contribution from an overactive tibialis anterior in one patient, whereas in another, it may be the overactivity of tibialis posterior [7].

This concept, namely, the identification of the muscle or muscles that contribute dynamically and statically to the UMN motor dysfunction, serves as a conceptual basis for this review. Simply put, identifying muscles that produce deforming maladaptive joint movements and postures statically and dynamically is an important endeavor in aiding clinical interpretation of gait dysfunction and in rationalizing subsequent treatment interventions.

Problems of movement control and limb deformities are common consequences of UMN syndrome. Dynamic EMG, gait, motion analysis, and diagnostic nerve blocks can frequently provide the necessary detailed information about specific muscle groups that will help guide decision making for selecting and defining a treatment strategy. Before selecting treatment interventions, the clinical team with the patient should explicitly develop functional goals and expectations.

Functional goals may be classified as type I symptomatic, type II - passive, or type III - active in nature. A symptomatic goal refers for example to clonus, flexor, or extensor spasms and pain among others as some of the targeted goals. Active function refers to a patient's direct use of the limb to carry out a functional activity. Passive function, on the other hand, refers to the passive manipulation of their limbs with their noninvolved limbs or through the assistance of caregivers to achieve functional ends.

\section{Table 3. Classification of UMN Syndrome Related Problems}

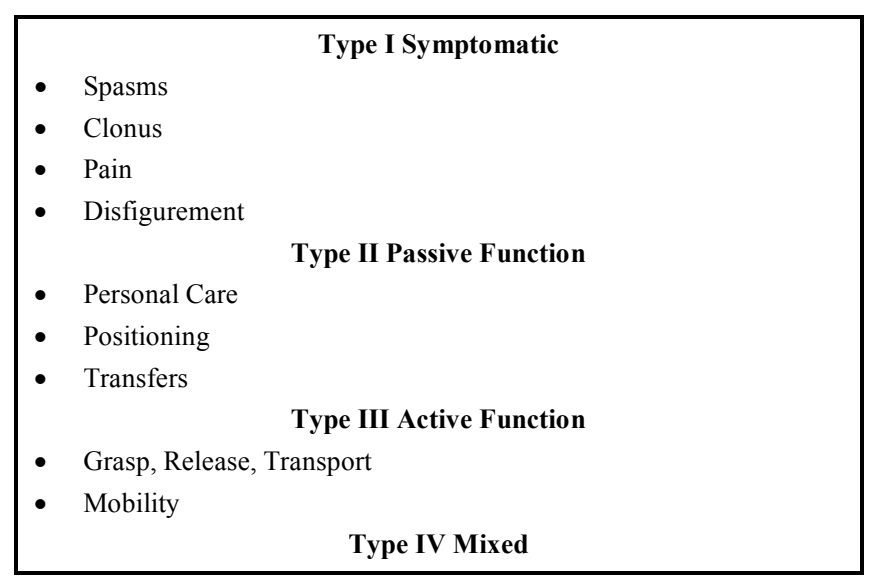

Identifying muscles with volitional capacity is crucial to the achievement of these goals. In broad terms, clinical evaluation focuses on the identification of several factors. Is their selective voluntary control of a given muscle? Is the muscle resistive to passive stretch? Does the muscle have fixed shortening (contracture)? In the Gait and Motor Control Analysis Laboratories, dynamic EMG is acquired and evaluated in reference to simultaneous measurements of joint motion (kinematics) and ground reaction forces (kinetics) obtained from force platforms. Kinetic, kinematic, and dynamic EMG data augment the clinician's ability to interpret whether voluntary function is present in a given muscle and whether that muscle behavior is activated dyssynergically (i.e. as an antagonist in movement).

Combined with clinical information, the laboratory measurements of muscle activation and function often provide the degree of detail and confidence necessary to optimize the rehabilitation interventions. In addition, evaluation before and after temporary diagnostic nerve or motor point blocks can help the clinician distinguish between obligatory and compensatory limb postures and gait patterns.

\section{GAIT}

The three main functional goals of human ambulation are to move from one place to another, to move safely, and to move efficiently. These three key goals are frequently compromised in the patient with residual from UMN syndrome. The great majority of patients will be able to perform limited ambulation, but they will often have problems because of inefficient movement strategies, the presence of pain or instability due to abnormal limb postures, and decreased safety. Some generalizations can be made about the gait of these patients. Ochi, et al. [8] reported on differences in temporo-spatial variables of locomotion among patients with residual stroke and traumatic brain injury. Decrease in walking velocity with a reduction in the duration of stance phase and impairment of weight bearing in the affected limb with an increase in the duration of stance time of the less affected limb.

From a functional perspective, gait deficiencies can be categorized with respect to the gait cycle. In the stance phase, an abnormal base of support can be caused by equinovarus, toe flexion, or ankle valgus.

Limb instability can occur due to knee buckling (sudden flexion) or hyperextension, which may result in knee joint pain. This may make walking unsafe, energy inefficient, and painful. During the swing phase, inadequate limb clearance caused, for example, by a stiff knee and inadequate limb advancement, may interfere with the safety and energy efficiency of walking.

\section{PATTERNS OF UMN DYSFUNCTION}

Because of space limitations, only the most common patterns of the seven UMN patterns of dysfunction in the lower limb have been selected for this review, and include (1) equinovarus foot, (2) hyperextended great toe, (3) stiff knee, (4) adducted (scissoring) thighs, and (5) flexed hip [7].

The first two patterns are considered to be problematic throughout the gait cycle. Stiff knee and adducted thigh are predominantly deviations of swing phase. The flexed hip is considered a stance-phase deviation. Potentially all the patterns influence translation or stability of the center of gravity in some way as described bellow.

\section{Equinovarus}

Equinovarus foot is the commonest UMN posture seen in the lower limb. The foot and ankle are turned down and in. 
Toe curling or toe clawing may coexist. The lateral border of the foot, particularly the fifth metatarsal head, is typically compressed against the floor or against the footrest of a wheelchair. Skin breakdown over the metatarsal head may develop from unrelieved pressure. Equinovarus is frequently maintained throughout stance phase, and inversion may increase, causing ankle instability as weight bearing is applied. Limited dorsiflexion during early and mid stance prevents the appropriate forward advancement of the tibia over the stationary foot, which frequently contributes to knee hyperextension.

Impairment in dorsiflexion range of motion in the late stance and pre-swing phases interferes with push-off and forward propulsion. During swing phase, equinus posture of the foot may result in a limb clearance problem, whereas the lack of adequate posture of the foot in stance phase may result in instability of the body as a whole. Under these circumstances, correction of this problem is essential, even for limited ambulation. A number of muscles may generate unbalanced forces with respect to the equinovarus pattern. Muscles that can potentially contribute to the equinovarus deformity include the tibialis anterior, tibialis posterior, long toe flexors, gastrocnemius, soleus, extensor hallucis longus, and the peroneus longus. Weak muscles may include peroneus longus and peroneus brevis in stance phase and the long toe extensors in swing phase.

The dynamic poly-EMG recordings of the above mentioned muscles in combination with the clinical examination provide a more detailed understanding of the genesis of this deformity. The recordings often demonstrate prolonged activation of the gastrocnemius and soleus complex and the long toe flexors as the commonest cause of plantar flexion. Occasionally, the gastrocnemius and soleus may activate differentially, and treatment interventions must take this into consideration. Inversion is the result of the overactivation of the tibialis posterior and/or anterior in combination with the gastrocnemius and soleus and, at times, the extensor hallucis longus also may contribute. If the tibialis posterior and anterior are both a factor in the varus deformity, a decision has to be made about which one of the two muscles is the main force.

Two approaches are possible for this differentiation. One is to use the joint powers obtained as part of the kinematic data in routine gait analysis. The second possibility is a diagnostic tibial nerve block with lidocaine. One has to be mindful that reducing the activation of the gastrocnemius soleus complex will tend to increase dorsiflexion and that tightness of the toe flexors usually becomes more apparent as a result of toe flexor tenodesis effect brought on by the increased dorsiflexion.

Based on dynamic poly-EMG correlated with clinical findings, the treatment of choice is chemodenervation of the tibialis posterior, gastrocnemius, soleus, and long toe flexors. When a contracture is evident, surgical intervention may need to be considered.

\section{Hyperextended Great Toe}

Hyperextended great toe is a deformity that is characterized by toe extension throughout the gait cycle, sometimes referred to as striatal toe or hitchhiker's toe. Ankle equinus and varus may accompany this foot deformity. When wearing shoes, the patient complains of pain at the tip of the toe and under the first metatarsal head during stance.

In gait, toe extension during early and mid stance complicates weight bearing. Thus, hyperextended great toe has an impact on translation of the center of gravity during stance-limb advancement. It also has an impact on center-ofgravity stability during stance phase loading and single-limb support. A number of muscles may generate unbalanced forces with respect to the hyperextended great toe pattern. Extensor hallucis longus provides a deforming force causing great toe hyperextension. A weak flexor hallucis longus may not be able to compensate and offset the extension force of the extensor hallucis longus. When equinovarus is present, analysis of the contributions of tibialis anterior, tibialis posterior, gastrocnemius, soleus, and the long toe flexors are taken into account as well. Chemodenervation of extensor hallucis longus can easily be achieved.

\section{Stiff Knee}

The stiff knee is a swing-phase deformity by definition. The knee is kept extended during preswing and initial swing, resulting in a reduction of the arc of motion, with its peak at $40 \circ$ (normal reference $60 \circ$ ). In addition, there may be delay in the timing of flexion and a concomitant reduction in hip flexion. Knee flexion during walking is generated by hip flexion. Reduced hip flexion moment may result in decreased knee flexion in swing phase. This deviation may cause foot drag. A stiff knee lengthens the limb and poses problems, particularly, during the pre-swing and swing phases of gait. Toe drag and early swing may cause tripping and falling if there is an associated equinovarus. The limb seems to be functionally longer because it remains extended throughout the swing phase. To achieve clearance of the floor during swing phase, the patient may attempt to compensate for this relative leg length discrepancy by ipsilateral limb circumduction, by hiking the pelvis, or by contralateral vaulting that consumes increased energy.

The effect of stiff knee includes an increase in the inertial load of the swinging limb (by virtue of its longer moment arm when the knee is extended compared with a flexed knee), thereby potentially influencing center-of-gravity translation during swing limb advancement. An extended knee may become problematic during swing-phase clearance and its associated effect on center-of-gravity stability. Frequently, a reduction is noted in the activation of iliopsoas, along with excessive activation of the rectus femoris, vastus intermedius, vastus medialis, vastus lateralis, and at times, excessive activation of the gluteus maximus. If an ankle equinus deformity is also present, a reduction in joint power generation and plantar flexion moment may result in further reduction of knee flexion.

Based on clinical and laboratory findings, chemodenervation to individual heads of the quadriceps may be considered. An overactive gluteus maximus in swing phase may act to restrain hip flexion and swing-limb advancement, resulting in a non swinging extended knee.

\section{Adducted (Scissoring) Thigh}

This deformity is characterized by adduction of the hip during the swing phase of locomotion. An adducted thigh 
results in a narrow base of support in stance phase, with potential impairment of balance. It also can interfere with limb clearance and advancement because the adducting swing-phase limb may collide with the stance limb. When adductor spasticity is complicated by hip flexor spasticity, toileting functions and perineal access are markedly hampered, and frequent repositioning of the patient sitting in a chair is required and usually does not work well. In walking. The adducted posturing at the end of swing phase generates a narrow base of support during stance, ultimately making upright balance precarious.

Dynamic poly-EMG recordings will demonstrate overactivation of the hip adductors, medial hamstrings, and pectineus. Weakness of the hip abductors and the iliopsoas may also contribute to this deformity because the patient may be attempting to use the hip adductors compensatory to advance the limb forward in swing phase.

It is essential to ascertain if the deformity is obligatory (the result of adductor spasticity) or compensatory (the result of weak hip flexors) because treatment will differ. A percutaneous temporary diagnostic obturator nerve block can be used to differentiate the role of hip adduction if the clinician is uncertain. Longer term interventions, such as percutaneous obturator nerve blocks, are easily carried out. After the intervention, aggressive stretching of the hip adductors and strengthening of the hip flexors and abductors is pursued.

\section{Flexed Hip}

The patient with excessive hip flexion experiences difficulty when standing up from a seated position during both phases of the gait cycle and potentially during positioning efforts for perineal care and sexual intimacy. In normal gait, the hip is flexed at initial contact but thereafter extends throughout stance phase.

The UMN pattern of hip flexion is defined as persistent hip flexion throughout stance. Severe hip flexion deformity may promote development of a knee flexion deformity.
When the limb with excessive hip flexion is in stance phase, forward advancement of the body over this limb is markedly restricted. The result is a shortened contralateral step. Excessive hip flexion also affects stability of the center of gravity by influencing single-limb support during stance.

Dynamic poly-EMG recordings of iliopsoas, pectineus, gluteus maximus, rectus femoris, and lumbar paraspinals should help in determining the contribution of the different muscles in the gait cycle. Overactive iliopsoas, rectus femoris, hip adductors, or weakness of the hip extensors and paraspinals may be evident. Interventions to reduce spasticity of the hip flexors (iliopsoas and rectus femoris), can be easily performed and followed by appropriate rehabilitation techniques.

\section{REFERENCES}

[1] Mayer N, Esquenazi A. Upper Limb Skin and Musculoskeletal Consequences of the Upper Motor Neuron Syndrome. In: Jankovic J, Albanese A, Atassi MZ, Dolly JO, Hallet M, Mayer NH, Eds. Philadelphia, USA: Botulinum Toxin: Therapeutic Clinical Practice and Science 2009; 131-47.

[2] Mayer N, Esquenazi A. Muscle overactivity and movement dysfunction in the upper motoneuron syndrome. Phys Med Rehabil Clin N Am 2003; 14: 855-83.

[3] Lance JW. Symposium synopsis. In: Feldman RG, Young RR Koella WP, Eds. Spasticity: Disordered motor control. Chicago: Yearbook Medical 1980; pp. 485-94.

[4] Walshe FMR. On certain tonic or postural reflexes in hemiplegia, with special reference to the so-called 'associated movements'. Brain 1923; 46: 1-37.

[5] Bourbonnais D. Quantification of upper limb synkinesis in hemiparetic subjects. Rehabilitation R \& D progress report 199495; 32: 118-9.

[6] Dewald JPA, Rymer WZ. Factors underlying abnormal posture and movement in spastic hemiparesis, In: Thilmann AF, Burke DJ, Rymer WZ, Eds. Spasticity: Mechanisms and Management. Berlin: Springer Verlag 1993; pp. 123-38.

[7] Mayer NH, Esquenazi A, Childers MK. Common patterns of clinical dysfunction. Muscle Nerve 1997; (Suppl 6): 21-33.

[8] Ochi F, Esquenazi A, Hirai B, Talaty M. Temporal-spatial feature of gait after traumatic brain injury. J Head Trauma Rehabil 1999; 14(2): 105-15. 